\title{
Novel evaluation of the two-pion contribution to the nucleon isovector form factors
}

\author{
M.A. Belushkin* and H.-W. Hamment \\ Helmholtz-Institut für Strahlen- und Kernphysik (Theorie), \\ Universität Bonn, Nußallee 14-16, D-53115 Bonn, Germany \\ Ulf-G. Meißner \\ Helmholtz-Institut für Strahlen- und Kernphysik (Theorie), \\ Universität Bonn, Nußallee 14-16, \\ D-53115 Bonn, Germany \\ and \\ Institut für Kernphysik (Theorie), \\ Forschungszentrum Jülich, \\ D-52425 Jülich, Germany
}

(Dated: February 2, 2008)

\begin{abstract}
We calculate the two-pion continuum contribution to the nucleon isovector spectral functions drawing upon the new high statistics measurements of the pion form factor by the CMD-2, KLOE, and SND collaborations. The general structure of the spectral functions remains unchanged, but the magnitude increases by about $10 \%$. Using the updated spectral functions, we calculate the contribution of the two-pion continuum to the nucleon isovector form factors and radii. We compare the isovector radii with simple $\rho$-pole models and illustrate their strong underestimation in such approaches. Moreover, we give a convenient parametrization of the result for use in future form factor analyses.

PACS numbers: 11.55.Fv, 13.40.Gp, 14.20.Dh
\end{abstract}

*Electronic address: belushki@itkp.uni-bonn.de

${ }^{\dagger}$ Electronic address: hammer@itkp.uni-bonn.de

${ }^{\ddagger}$ Electronic address: meissner@itkp.uni-bonn.de 


\section{INTRODUCTION}

The electromagnetic form factors of the nucleon offer a unique window on strong interaction dynamics over a wide range of momentum transfers [1, 2]. At small momentum transfers, one is only sensitive to the gross properties of the nucleon like the charge and magnetic moment, while at high momentum transfers one will be able to resolve aspects of the quark substructure of the nucleon as described by QCD. The form factors encode both perturbative and non-perturbative aspects of QCD such as the nucleon radii, vector meson coupling constants, and the asymptotic behavior at large momentum transfer. Moreover, their detailed understanding is important for a wide variety of experiments ranging from the strange vector form factors of the nucleon 3] to Lamb shift measurements in atomic hydrogen [4].

It has been known for a long time that the pion plays an important role in the longrange structure of the nucleon [5]. This connection was made more precise using dispersion theory in the 1950's 6, 7]. Subsequently, Frazer and Fulco have written down partial wave dispersion relations that relate the nucleon electromagnetic structure to pion-nucleon $(\pi N)$ scattering and predicted the existence of the $\rho$ resonance [8, 9]. Despite of this success, the central role of the $2 \pi$ continuum in the isovector spectral function has often been ignored in vector-meson dominance analyses of the electromagnetic form factors of the nucleon where the $\rho$ was approximated by a simple pole. In 1975, Höhler and Pietarinen pointed out that this omission leads to a gross underestimate of the isovector radii of the nucleon [10]. Höhler et al. first perfomed a consistent dispersion analysis of the electromagnetic form factors of the nucleon [11] including the $2 \pi$ continuum derived from the pion form factor and $\pi N$ scattering data [12]. In the mid-nineties, this analysis has been updated [13] and extended to include data in the time-like region [14]. Recently, the new precise data for the neutron electric form factor have been included as well [15].

Using chiral perturbation theory (CHPT), the long-range pionic structure of the nucleon can be connected to the Goldstone boson dynamics of QCD [16]. The nonresonant part of the $2 \pi$ continuum is in excellent agreement with the phenomenological analysis [17] and the $\rho$-meson contribution can be included as well [18, 19, 20]. It is well known that vector mesons play an important role in the electromagnetic structure of the nucleon, see e.g. 8, 10, 21, 22, 23, 24, 25], and the remaining contributions to the spectral function have usually been approximated by vector meson resonances.

A new twist to this picture was recently given in Ref. [26], where the form factor data were interpreted based on a phenomenological fit with an ansatz for the pion cloud using the old idea that the proton can be thought of as virtual neutron-positively charged pion pair. A very long-range contribution to the charge distribution in the Breit frame extending out to about $2 \mathrm{fm}$ was found and attributed to the pion cloud. While naively the pion Compton wave length is of this size, these findings are indeed surprising if compared with the "pion cloud" contribution due to the $2 \pi$ contribution for the isovector spectral functions of the nucleon form factors, which can be obtained from unitarity or chiral perturbation theory. As was shown in Ref. [27], these latter contributions to the long-range part of the nucleon structure are much more confined in coordinate space and agree well with earlier (but less systematic) calculations based on chiral soliton models, see e.g. [28]. Therefore it remains to be shown how to reconcile the findings of Ref. [26], based on a global fit to all nucleon form factors, with the results of dispersion analysis and chiral perturbation theory.

The CMD-2 [29], KLOE [30], and SND 31] collaborations have recently remeasured the 
pion form factor with high statistics and found significant deviations from earlier measurements at the $\rho$-resonance peak. Moreover, the three measurements also show discrepancies among each other. In light of the importance of the $2 \pi$ continuum for the electromagnetic structure of the nucleon, it appears worthwhile to recalculate the $2 \pi$-continuum contribution to the isovector spectral function of the nucleon using the new high statistics data and estimate the errors that arise from the discrepancy among the experiments. Moreover, a new analysis will help to better understand the nature and range of the pion cloud of the nucleon.

\section{NUCLEON FORM FACTORS}

First, we collect some basic definitions. The nucleon electromagnetic (EM) form factors are defined by the nucleon matrix element of the electromagnetic current,

$$
\left\langle N\left(p^{\prime}\right)\left|J_{\mu}^{\mathrm{EM}}\right| N(p)\right\rangle=\bar{u}\left(p^{\prime}\right)\left[\gamma^{\mu} F_{1}(t)+\frac{i}{2 m} \sigma^{\mu \nu}\left(p^{\prime}-p\right)_{\nu} F_{2}(t)\right] u(p),
$$

with $t=\left(p^{\prime}-p\right)^{2}=q^{2}<0$ the invariant momentum transfer squared, and $m$ the nucleon mass. $F_{1}(t)$ and $F_{2}(t)$ are the Dirac and the Pauli form factors, respectively. They are normalized at $t=0$ to the charge $\left(F_{1}\right)$ and anomalous magnetic moment $\left(F_{2}\right)$. It is convenient to work in the isospin basis and to decompose the nucleon form factors into isoscalar $(S)$ and isovector $(V)$ parts,

$$
F_{i}^{S}=\frac{1}{2}\left(F_{i}^{p}+F_{i}^{n}\right), \quad F_{i}^{V}=\frac{1}{2}\left(F_{i}^{p}-F_{i}^{n}\right), \quad i=1,2,
$$

where $p(n)$ denotes the proton (neutron). The experimental data are usually given for the Sachs form factors, which are linear combinations of $F_{1}$ and $F_{2}$

$$
G_{E}^{I}(t)=F_{1}^{I}(t)-\tau F_{2}^{I}(t), \quad G_{M}^{I}(t)=F_{1}^{I}(t)+F_{2}^{I}(t), \quad I=S, V,
$$

where $\tau=-t /\left(4 m^{2}\right)$. In the Breit frame, where the energy transfer of the virtual photon vanishes, $G_{E}$ and $G_{M}$ may be interpreted as the Fourier transforms of the charge and magnetization distributions in coordinate space, respectively.

The analysis of the nucleon electromagnetic form factors proceeds most directly through the spectral representation given by ${ }^{1}$

$$
F_{i}^{I}(t)=\frac{1}{\pi} \int_{t_{0}^{I}}^{\infty} \frac{\operatorname{Im} F_{i}^{I}\left(t^{\prime}\right) d t^{\prime}}{t^{\prime}-t}, \quad i=1,2, \quad I=S, V,
$$

where the corresponding thresholds are given by $t_{0}^{S}=\left(3 M_{\pi}\right)^{2}$ and $t_{0}^{V}=\left(2 M_{\pi}\right)^{2}$, respectively and $M_{\pi}$ is the pion mass. The imaginary part entering Eq. (44) can be obtained from a

\footnotetext{
${ }^{1}$ We work here with unsubtracted dispersion relations. Since the normalizations of all the form factors are known, one could also work with once-subtracted dispersion relations. For the topic studied here, this is of no relevance.
} 
spectral decomposition [7]. For this purpose it is convenient to consider the EM current matrix element in the time-like region,

$$
J_{\mu}=\left\langle N(p) \bar{N}(\bar{p})\left|J_{\mu}^{\mathrm{em}}\right| 0\right\rangle=\bar{u}(p)\left[F_{1}(t) \gamma_{\mu}+\frac{i}{2 m} \sigma_{\mu \nu}(p+\bar{p})^{\nu} F_{2}(t)\right] v(\bar{p}),
$$

where $p, \bar{p}$ are the momenta of the nucleon-antinucleon pair created by the current $J_{\mu}^{\mathrm{em}}$. The four-momentum transfer in the time-like region is $t=(p+\bar{p})^{2}>0$. Using the LSZ reduction formalism, the imaginary part of the form factors is obtained by inserting a complete set of intermediate states as [7]

$$
\operatorname{Im} J_{\mu}=\frac{\pi}{Z}(2 \pi)^{3 / 2} \mathcal{N} \sum_{\lambda}\left\langle p\left|\bar{J}_{N}(0)\right| \lambda\right\rangle\left\langle\lambda\left|J_{\mu}^{\mathrm{em}}\right| 0\right\rangle v(\bar{p}) \delta^{4}\left(p+\bar{p}-p_{\lambda}\right),
$$

where $\mathcal{N}$ is a nucleon spinor normalization factor, $Z$ is the nucleon wave function renormalization, and $\bar{J}_{N}(x)=J_{N}^{\dagger}(x) \gamma_{0}$ with $J_{N}(x)$ a nucleon source. The states $|\lambda\rangle$ are asymptotic states of momentum $p_{\lambda}$. They carry the same quantum numbers as the current $J_{\mu}^{\mathrm{em}}$ : $I^{G}\left(J^{P C}\right)=0^{-}\left(1^{--}\right)$for the isoscalar component and $I^{G}\left(J^{P C}\right)=1^{+}\left(1^{--}\right)$for the isovector component of $J_{\mu}^{\mathrm{em}}$. Furthermore, they have no net baryon number.

For the isoscalar current the lowest mass states are: $3 \pi, 5 \pi, \ldots$; for the isovector part they are: $2 \pi, 4 \pi, \ldots$. Because of $G$-parity, states with an odd number of pions only contribute to the isoscalar part, while states with an even number contribute to the isovector part. Associated with each intermediate state is a cut starting at the corresponding threshold in $t_{0}$ and running to infinity. As a consequence, the spectral function $\operatorname{Im} F(t)$ is different from zero along the cut from $t_{0}$ to $\infty$ with $t_{0}=4(9) M_{\pi}^{2}$ for the isovector (isoscalar) case. Using Eqs. (56), the spectral functions for the form factors can in principle be constructed from experimental data. In practice, this program can only be carried out for the lowest-mass two-particle intermediate states $(2 \pi$ and $K \bar{K})$ 12, 32, 33].

The longest-range (and therefore most important at low momentum transfer) pion cloud contribution comes from the $2 \pi$ intermediate state in the isovector form factors. This contribution was first constructed from the pion form factor and $\pi N$ scattering data by Höhler and Pietarinen [12].

\section{TWO-PION CONTINUUM}

In this paper, we re-evaluate the $2 \pi$ contribution in a model-independent way using the latest experimental data for the pion form factor from CMD-2 29], KLOE [30], and SND 31]. We follow Ref. [34] and express the $2 \pi$ contribution to the the isovector spectral functions in terms of the pion charge form factor $F_{\pi}(t)$ and the $\mathrm{P}$-wave $\pi \pi \rightarrow \bar{N} N$ amplitudes $f_{ \pm}^{1}(t)$. The $2 \pi$ continuum is expected to be the dominant contribution to the isovector spectral function from threshold up to masses of about $1 \mathrm{GeV}$ [34]. Here, we use the expressions

$$
\begin{aligned}
\operatorname{Im} G_{E}^{V}(t) & =\frac{q_{t}^{3}}{m \sqrt{t}} F_{\pi}(t)^{*} f_{+}^{1}(t), \\
\operatorname{Im} G_{M}^{V}(t) & =\frac{q_{t}^{3}}{\sqrt{2 t}} F_{\pi}(t)^{*} f_{-}^{1}(t),
\end{aligned}
$$

where $q_{t}=\sqrt{t / 4-M_{\pi}^{2}}$. The imaginary parts of the Dirac and Pauli Form factors can be obtained using Eq. (3). The $\mathrm{P}$-wave $\pi \pi \rightarrow \bar{N} N$ amplitudes $f_{ \pm}^{1}(t)$ are tabulated in 


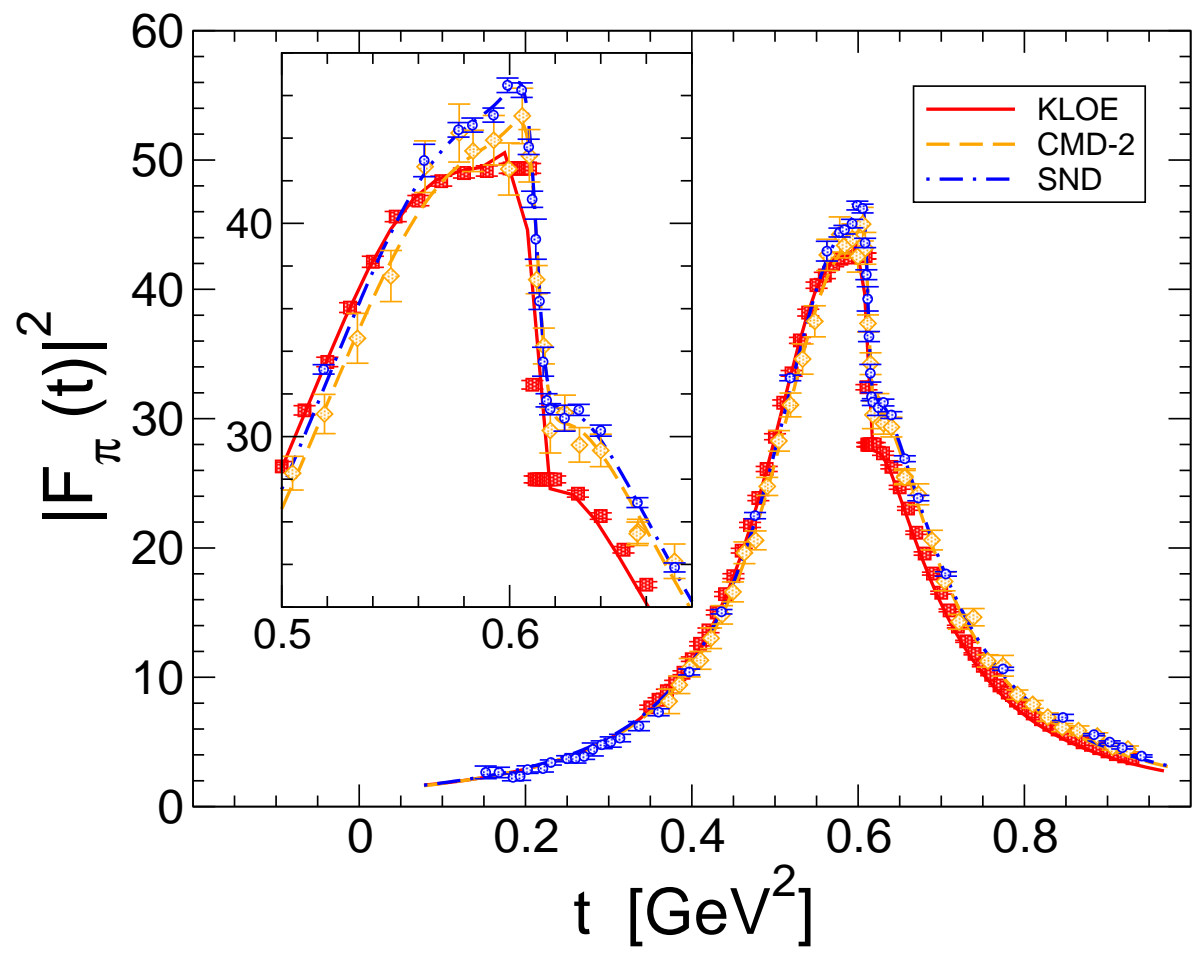

FIG. 1: The pion electromagnetic form factor in the time-like region as a function of the momentum transfer $t$. The diamonds, squares, and circles show the high statistics data from the CMD-2 29], KLOE [30], and SND 31] collaborations, respectively. The dashed, solid, and dash-dotted lines are our model parametrizations. The inset shows the discrepancy in the resonance region in more detail.

Ref. [34]. (See also Ref. [35] for an unpublished update that is consistent with Ref. [34].) We stress that the representation of Eq. (77) gives the exact isovector spectral functions for $4 M_{\pi}^{2} \leq t \leq 16 M_{\pi}^{2}$, but in practice holds up to $t \simeq 50 M_{\pi}^{2}$. Since the contributions from $4 \pi$ and higher intermediate states is small up to $t \simeq 50 M_{\pi}^{2}, F_{\pi}(t)$ and the $f_{ \pm}^{1}(t)$ share the same phase in this region and the two quantities can be replaced by their absolute values. ${ }^{2}$

The updated pion form factor is shown in Fig. 1. The diamonds, squares, and circles show the high statistics data from the CMD-2 [29], KLOE 30], and SND 31] collaborations, respectively. The dashed, solid, and dash-dotted lines are our model parametrizations which are of the Gounaris-Sakurai type [13, 22]. The form factor shows a pronounced $\rho$ - $\omega$ mixing in the vicinity of the $\rho$-peak. There are discrepancies between the three experimental data sets for the pion form factor [31]. The discrepancies in the $\rho$-resonance region are shown in more detail in the inset of Fig. 1. Since we are not in the position to settle this experimental problem, we will take the three data sets at face value. We will evaluate the $2 \pi$ continuum given by Eq. (17) for all three sets and estimate the errors from the discrepancy between the sets.

Using the new high statistics pion form factor data [29, 30, 31] and the amplitudes

\footnotetext{
${ }^{2}$ We note that representation of Eq. (7) is most useful for our purpose. The manifestly real functions $J_{ \pm}(t)=f_{ \pm}^{1}(t) / F_{\pi}(t)$ also tabulated in Ref. 34] contain assumptions about the pion form factor which leads to inconsistencies when used together with the updated $F_{\pi}(t)$.
} 


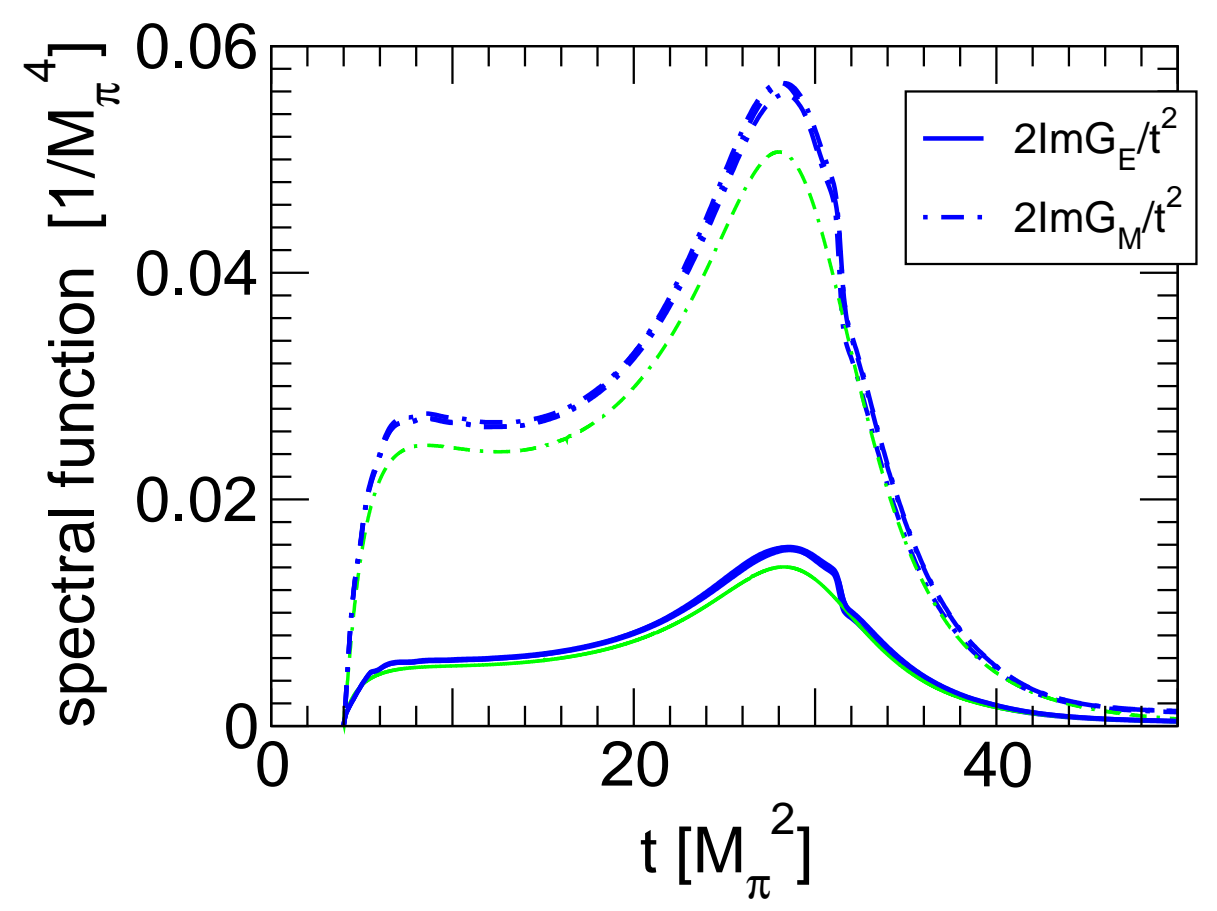

FIG. 2: The $2 \pi$ spectral function using the new high statistics data for the pion form factor 29, 30, 31]. The spectral functions weighted by $1 / t^{2}$ are shown for $G_{E}$ (solid line) and $G_{M}$ (dash-dotted line). The previous results by Höhler et al. 34] (without $\rho$ - $\omega$ mixing) are shown for comparison by the gray/green lines.

$f_{ \pm}^{1}(t)$ tabulated in Ref. [34], we obtain the spectral functions shown in Fig. 2. We show the spectral functions weighted by $1 / t^{2} G_{E}$ (solid line) and $G_{M}$ (dash-dotted line). The previous results by Höhler et al. [34] (without $\rho$ - $\omega$ mixing) are given for comparison by the gray/green lines. The general structure of the two evaluations is the same, but there is a difference in magnitude of about $10 \%$. The difference between the three data sets for the pion form factor is very small and indicated by the line thickness. The difference in the form factors is largest in the $\rho$-peak region (cf. Fig. (1), but this region is suppressed by the $\pi \pi \rightarrow \bar{N} N$ amplitudes $f_{ \pm}^{1}(t)$ which show a strong fall-off as $t$ increases.

The spectral functions have two distinct features. First, as already pointed out in [8], they contain the important contribution of the $\rho$-meson with its peak at $t \simeq 30 M_{\pi}^{2}$. Second, on the left shoulder of the $\rho$, the isovector spectral functions display a very pronounced enhancement close to the two-pion threshold. This is due to the logarithmic singularity on the second Riemann sheet located at $t_{c}=4 M_{\pi}^{2}-M_{\pi}^{4} / m^{2}=3.98 M_{\pi}^{2}$, very close to the threshold. This pole comes from the projection of the nucleon Born graphs, or in modern language, from the triangle diagram. If one were to neglect this important unitarity correction, one would severely underestimate the nucleon isovector radii [10]. In fact, precisely the same effect is obtained at leading one-loop accuracy in relativistic chiral perturbation theory 36, 37. This topic was further elaborated on in the framework of heavy baryon CHPT [17, 20] and in a covariant calculation based on infrared regularization [18]. Thus, the most important $2 \pi$ contribution to the nucleon form factors can be determined by using either unitarity or CHPT (in the latter case, of course, the $\rho$ contribution is not included).

The importance of the correct $2 \pi$-continuum contribution for the nucleon isovector radii 


\begin{tabular}{|l|c|c|}
\hline Ref. & $\left\langle r^{2}\right\rangle_{1}^{V}\left[\mathrm{fm}^{2}\right]$ & $\left\langle r^{2}\right\rangle_{2}^{V}\left[\mathrm{fm}^{2}\right]$ \\
\hline this work & $0.32 \ldots 0.33$ & $1.77 \ldots 1.80$ \\
Ref. [20] & 0.19 & 1.10 \\
Ref. [38] (i) & 0.19 & 0.96 \\
Ref. [38] (ii) & 0.27 & 1.38 \\
\hline
\end{tabular}

TABLE I: Comparison of the $2 \pi$-continuum contribution to the Dirac and Pauli isovector radii of the nucleon (first line) to three different $\rho$-pole models from Refs. [20, 38]. The given range indicates the error introduced by the different pion form factor data sets.

is illustrated in Table 【. We compare the contribution of the full $2 \pi$ continuum and various $\rho$-pole parametrizations to the Dirac and Pauli isovector radii of the nucleon [10]:

$$
\left\langle r^{2}\right\rangle_{i}^{V}=\frac{6}{\pi} \int_{4 M_{\pi}^{2}}^{50 M_{\pi}^{2}} \frac{\operatorname{Im} F_{i}^{V}(t)}{t^{2}} d t, \quad i=1,2 .
$$

The first line shows the contribution of the full $2 \pi$ continuum from this work to the nucleon isovector radius. The given range indicates the error introduced by the different pion form factor data sets. The second line shows the $\rho$-pole parametrization used in Ref. [20]. The third and fourth lines show the $\rho$-pole contribution from Ref. 38] excluding $(i)$ and including $(i i)$ an approximate $2 \pi$ continuum, respectively. It is obvious that the simple $\rho$-pole parametrizations from lines two and three underestimate the full contribution by about 30$40 \%$ depending on the form factor. The approximate $2 \pi$ continuum from Ref. 38 in the fourth line comes fairly close for the Dirac form factor but still underestimates the Pauli radius by about $20 \%$.

Inserting the spectral functions into the dispersion relation Eq. (4), we obtain the $2 \pi$ contribution to the nucleon isovector form factors. The contribution of the spectral function in the region $t \geq 50 M_{\pi}^{2}$ is very small and set to zero in this evaluation. The results for the form factors can be fitted by an expression of the form [11, 13.

$$
F_{i}^{V}(t)=\frac{a_{i}+b_{i}\left(1-t / c_{i}\right)^{-2 / i}}{2\left(1-t / d_{i}\right)}, \quad i=1,2,
$$

where $a_{i}, b_{i}, c_{i}$, and $d_{i}$ are constants. Averaging the results for the three different pion form factor data sets [29, 30, 31], the values of the constants are $a_{1}=1.10788, b_{1}=0.109364$, $c_{1}=0.36963, d_{1}=0.553034, a_{2}=5.724253, b_{2}=1.111128, c_{2}=0.27175$, and $d_{2}=0.611258$. The errors in these constants are of the order $4 \%$ or less. Using the parametrization from Eq. (9), the $2 \pi$ contribution to the isovector form factors can easily be included in any form factor analysis. It is fixed by the pion form factor and $\pi N$ data and contains no free parameters.

\section{SUMMARY \& CONCLUSION}

In this letter, we have presented a novel analysis of the $2 \pi$ contribution to the nucleon isovector spectral function using the new high statistics data of the pion form factor by the CMD-2 [29], KLOE 30], and SND [31] collaborations. The difference in the spectral function between the three data sets for the pion form factor is very small. The spectral function 
displays the contribution of the $\rho$ peak around $t \simeq 30 M_{\pi}^{2}$ and the pronounced enhancement close to the two-pion threshold at $t=4 M_{\pi}^{2}$ from the logarithmic singularity on the second Riemann sheet. The magnitude of the spectral function increases by about $10 \%$ compared to the previous analyses.

The conclusions of Ref. [27] about the possibility of a long-range pion cloud remain unaffected by this change in magnitude: a long-range pion cloud extending as far as $2 \mathrm{fm}$ is not compatible with what is known about the $2 \pi$ contribution to the nucleon isovector spectral function. We note that this conclusion might have to be modified if the highermass pion-continua $(3 \pi, 4 \pi, \ldots)$ show a significant threshold enhancement similar to the $2 \pi$ continuum. Given the current state of knowledge, however, this appears unlikely. In Ref. [17], the threshold behavior of the $3 \pi$ continuum was explicitly calculated in heavy baryon ChPT and no enhancement was found. Moreover, the inelasticity from four pions in $\pi \pi$ scattering and four-pion production in $e^{+} e^{-}$annihilation at low momentum transfer are known to be small [34, 39, 40].

Finally, we have calculated the resulting contribution to the nucleon isovector form factors and given a convenient parametrization of the result. This contribution is fixed from the pion form factor and $\pi N$-scattering data and contains no free parameters. It can easily be used as an independent input in future form factor analyses. This will reduce the number of free parameters and ensure the correct spectral function on the nearest part of the cut in the time-like region. A new dispersion-theoretical analysis of the nucleon form factors using the updated $2 \pi$ continuum is in preparation [41].

\section{Acknowledgments}

We thank A. Denig for providing the KLOE data for the pion form factor and the referee for pointing out the CMD-2 and SND measurements. This work was supported in part by the EU Integrated Infrastructure Initiative Hadron Physics under contract number RII3CT-2004-506078 and the Deutsche Forschungsgemeinschaft through funds provided to the SFB/TR 16 "Subnuclear structure of matter".

[1] H. Gao, Int. J. Mod. Phys. E 12 (2003) 1 [Erratum-ibid. E 12 (2003) 567] arXiv:nucl-ex/0301002.

[2] C. E. Hyde-Wright and K. de Jager, Ann. Rev. Nucl. Part. Sci. 54 (2004) 217 arXiv:nucl-ex/0507001.

[3] D. H. Beck and B. R. Holstein, Int. J. Mod. Phys. E 10 (2001) 1 arXiv:hep-ph/0102053.

[4] Th. Udem et al., Phys. Rev. Lett. 79 (1997) 2646.

[5] H. Fröhlich, W. Heitler and N. Kemmer, Proc. Roy. Soc. A 166 (1938) 155.

[6] G.F. Chew, R. Karplus, S. Gasiorowicz, and F. Zachariasen, Phys. Rev. 110 (1958) 265.

[7] P. Federbush, M.L. Goldberger, and S.B. Treiman, Phys. Rev. 112 (1958) 642.

[8] W. R. Frazer and J. R. Fulco, Phys. Rev. Lett. 2 (1959) 365.

[9] W. R. Frazer and J. R. Fulco, Phys. Rev. 117 (1960) 1609.

[10] G. Höhler and E. Pietarinen, Phys. Lett. B 53 (1975) 471.

[11] G. Höhler et al., Nucl. Phys. B 114 (1976) 505.

[12] G. Höhler and E. Pietarinen, Nucl. Phys. B 95 (1975) 210. 
[13] P. Mergell, U.-G. Meißner and D. Drechsel, Nucl. Phys. A 596 (1996) 367 arXiv:hep-ph/9506375.

[14] H.-W. Hammer, U.-G. Meißner and D. Drechsel, Phys. Lett. B 385 (1996) 343 arXiv:hep-ph/9604294.

[15] H.-W. Hammer and U.-G. Meißner, Eur. Phys. J. A 20 (2004) 469 arXiv:hep-ph/0312081.

[16] V. Bernard, N. Kaiser and U.-G. Meißner, Int. J. Mod. Phys. E 4 (1995) 193 arXiv:hep-ph/9501384.

[17] V. Bernard, N. Kaiser and U.-G. Meißner, Nucl. Phys. A 611 (1996) 429 arXiv:hep-ph/9607428.

[18] B. Kubis and U.-G. Meißner, Nucl. Phys. A 679 (2001) 698 arXiv:hep-ph/0007056.

[19] B. Kubis and U.-G. Meißner, Eur. Phys. J. C 18 (2001) 747 arXiv:hep-ph/0010283.

[20] N. Kaiser, Phys. Rev. C 68 (2003) 025202 arXiv:nucl-th/0302072.

[21] J. J. Sakurai, Ann. Phys. (NY) 11 (1960) 1.

[22] G. J. Gounaris and J. J. Sakurai, Phys. Rev. Lett. 21 (1968) 244.

[23] M. Gari and W. Krümpelmann, Z. Phys. A 322 (1985) 689.

[24] E. L. Lomon, Phys. Rev. C 64 (2001) 035204 arXiv:nucl-th/0104039.

[25] S. Dubnicka, A. Z. Dubnickova and P. Weisenpacher, J. Phys. G 29 (2003) 405 arXiv:hep-ph/0208051.

[26] J. Friedrich and T. Walcher, Eur. Phys. J. A 17 (2003) 607 arXiv:hep-ph/0303054.

[27] H.-W. Hammer, D. Drechsel and U.-G. Meißner, Phys. Lett. B 586 (2004) 291 arXiv:hep-ph/0310240.

[28] U.-G. Meißner, Phys. Rept. 161 (1988) 213.

[29] R. R. Akhmetshin et al. [CMD-2 Collaboration], arXiv:hep-ex/9904027, Phys. Lett. B 527 (2002) 161 arXiv:hep-ex/0112031, Phys. Lett. B 578 (2004) 285 arXiv:hep-ex/0308008.

[30] A. Aloisio et al. [KLOE Collaboration], Phys. Lett. B 606 (2005) 12 arXiv:hep-ex/0407048.

[31] M. N. Achasov et al., arXiv:hep-ex/0506076.

[32] H.-W. Hammer and M. J. Ramsey-Musolf, Phys. Rev. C 60 (1999) 045205 [Erratum-ibid. C 62 (2000) 049903] arXiv:hep-ph/9812261.

[33] H.-W. Hammer and M. J. Ramsey-Musolf, Phys. Rev. C 60 (1999) 045204 [Erratum-ibid. C 62 (2000) 049902] arXiv:hep-ph/9903367.

[34] G. Höhler, "Pion-Nucleon Scattering", Landolt-Börnstein Vol. I/9b, ed. H. Schopper, Springer, Berlin, 1983.

[35] E. Pietarinen, "A calculation of $\pi \pi \rightarrow N \bar{N}$ amplitudes in the pseudophysical region", University of Helsinki Preprint Series in Theoretical Physics, HU-TFT-17-77, unpublished.

[36] J. Gasser, M. E. Sainio and A. Svarc, Nucl. Phys. B 307 (1988) 779.

[37] U.-G. Meißner, Int. J. Mod. Phys. E 1 (1992) 561.

[38] F. Iachello, Eur. Phys. J. A 19 (2004) SUPPL129 [FizikaB 13 (2004) 13].

[39] J. Gasser and U.-G. Meißner, Nucl. Phys. B 357 (1991) 90.

[40] G. Ecker and R. Unterdorfer, Eur. Phys. J. C 24 (2002) 535 arXiv:hep-ph/0203075.

[41] M.A. Belushkin, H.-W. Hammer, and U.-G. Meißner, in preparation. 